\title{
Some Basic Microwave Phase Shift Equations
}

\author{
Robert W. Beatty \\ Contribution from the Radio Standards Laboratory, National Bureau of Standards, Boulder, Colo.
}

(Received November 14, 1963)

\begin{abstract}
The phase differences between terminal variables (voltage, current, or traveling wave types) at the output with respect to similar ones at the input of a 2-port are expressed in terms of the scattering coefficients of the 2-port and of the reflection coefficients of the system into which it is inserted.

A variety of phase shifts may be defined for a given 2-port, depending upon which of the terminal variables are considered, whether or not generator and load reflection coefficients are assumed to vanish, and in which direction the 2-port has been inserted into the system.

It is shown that a reasonable choice for one of the two "characteristic" phase shifts of a 2-port is $\psi_{21}$, the argument of $S_{21}$, one of the scattering coefficients. It follows that the other "characteristic" phase shift is $\psi_{12}$. The corresponding change in characteristic phase shift for variable phase shifters is the change in either $\psi_{21}$ or $\psi_{12}$ (whichever is appropriate) from the initial to the final setting.

Ideal phase shifters are discussed, and expressions for the change in output level of variable phase shifters are given. The importance of using a nonreflecting system in phase shift measurements is emphasized.
\end{abstract}

\section{Introduction}

The measurement of the phase shifting properties of microwave devices has received relatively little attention, but is becoimng more important. One cannot find a reference to microwave phase shift measurements in any of the well-known texts on microwave measurements that have been published to date. However, evidences of increasing interest include the recent development by commercial organizations of several different swept frequency systems for microwave phase shift measurements. In addition, papers on this subject have been surveyed in a recent article by R. A. Sparks [1963].

It seems appropriate at this time to examine concepts of microwave phase shift in order to clearly understand what types of phase shift may occur and how they may be described in a quantitative way.

Specifically, it is proposed in this paper to present expressions relating the phase shifts associated with a) 2-port to the scattering coefficients of that 2-port and the reflection coefficients of the system in which the 2-port is connected.

\section{General}

Several types of phase shift of 2-ports may be considered, as will be shown with reference to figure 1.

The usefulness of the different types of phase shift considered will depend upon what types of

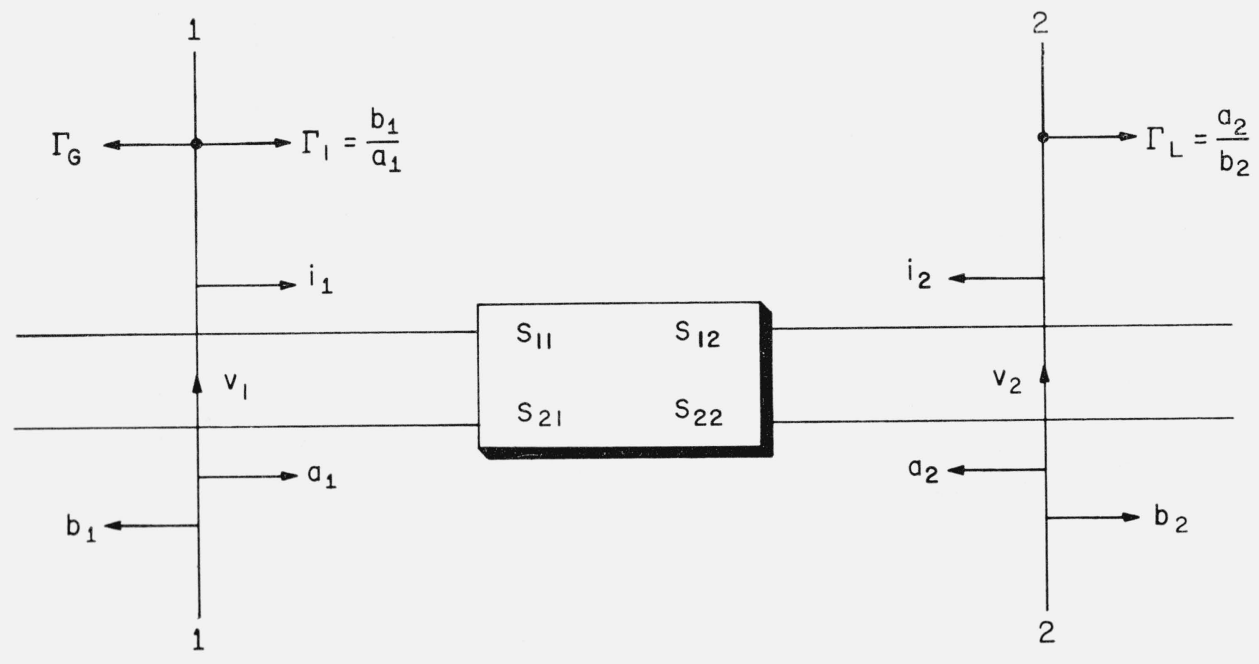

Figure 1. Representation of a 2-port, showing two sets of terminal variables. 
detectors are used in a measurement of phase difference and whether they respond to wave amplitudes or generalized voltage and current. (An electric field probe in a slotted line would respond to $v$, for example.)

In figure 1, the terminal surfaces $1-1$, and $2-2$, in the waveguide leads of the 2-port, are the places where the complex amplitudes $a$ and $b$ of the incident and emergent voltage waves, and $v$ and $i$, the generalized voltage and current, [Kerns, 1949] are to be considered. The assumptions inherent in this representation of a 2 -port, such as single-mode propagation in the lossless waveguide leads have been set forth by D. M. Kerns [1949 and 1951].

The relationship between the two sets of terminal variables is as follows

$$
\left.\begin{array}{l}
v=a+b \\
Z_{0} i=a-b
\end{array}\right\} .
$$

The amplitude $b_{G}$ of the generator wave, $a_{1}, b_{1}$, and $\Gamma_{G}$ are related by the following equation:

$$
a_{1}=b_{G}+b_{1} \Gamma_{G} .
$$

One can consider the phase shift of the 2-port to be the difference in phase between $v_{2}$ and $v_{1}$, between $i_{2}$ and $i_{1}$, between $b_{2}$ and $a_{1}$, or between $b_{2}$ and $b_{G}$, for example. "The phase shift of a 2-port" may, therefore, be a misleading and ambiguous expression, since it seems to imply either that there is only one, or that all phase shifts of a given 2-port are the same. In the following, equations will be given to show how these various phase shifts will differ in general, and under what conditions some of them may be the same.

The equations will be given for the case of a linear 2-port (which may be nonreciprocal) inserted into a system in such a direction that a generator feeds arm 1 and the load terminates arm 2. For simplicity, the symbols such as $\psi_{v}$ for phase shift do not indicate that this direction has been chosen. One can easily obtain the corresponding phase shift for the opposite direction of energy flow thru the 2-port by interchanging subscripts 1 and 2 in the equations.

If the phase shifter is a reciprocal one, the condition $Z_{01} S_{21}=Z_{02} S_{12}$ will hold, where $Z_{01}$ and $Z_{02}$ are the characteristic impedances of the waveguide leads 1 and 2 of the 2-port. Very often, $Z_{01}=Z_{02}$, and the reciprocity condition is written $S_{21}=S_{12}$. This condition may be substituted into the phase shift equations in order to reduce the number of variables by one for a reciprocal 2-port.

\section{Phase Shift Equations}

The derivation of the following expressions for phase shift will not be given, as they follow from straightforward algebraic manipulation of eqs (1) and (2) and the scattering equations of the 2-port.

$$
\left.\begin{array}{l}
b_{1}=S_{11} a_{1}+S_{12} a_{2} \\
b_{2}=S_{21} a_{1}+S_{22} a_{2}
\end{array}\right\} .
$$

\subsection{Phase Shift of $v$}

The phase shift of $v$, denoted by $\psi_{0}$, may be written as follows

$$
\psi_{v}=\arg \frac{v_{2}}{v_{1}}=\arg \frac{S_{21}\left(1+\Gamma_{L}\right)}{\left(1+S_{11}\right)\left(1-S_{22} \Gamma_{L}\right)+S_{12} S_{21} \Gamma_{L}} .
$$

This phase shift depends upon the reflection coefficient of the load as well as upon the characteristics of the 2-port. When the load is nonreflecting,

$$
\left[\psi_{v}\right]_{\Gamma_{L}=0}=\arg \frac{S_{21}}{1+S_{11}} .
$$

If one employs the impedance matrix instead of the scattering matrix of the 2-port, the equations corresponding to (4) and (5) are

$$
\psi_{v}=\arg \frac{Z_{21} Z_{L}}{Z_{11}\left(Z_{22}+Z_{L}\right)-Z_{12} Z_{21}},
$$

and

$$
\left[\psi_{v}\right]_{Z_{L}=1}=\arg \frac{Z_{21}}{Z_{11}\left(Z_{22}+1\right)-Z_{12} Z_{21}},
$$

where normalized impedances are used.

For an open-circuited phase shifter,

$$
\left[\psi_{v}\right]_{Z_{L}=\infty}=\arg \frac{Z_{21}}{Z_{11}}
$$

\subsection{Phase Shift of $i$}

Proceeding in a similar way,

$$
\psi_{i}=\arg \frac{-i_{2}}{i_{1}}=\arg \frac{S_{21}\left(1-\Gamma_{L}\right)}{\left(1-S_{11}\right)\left(1-S_{22} \Gamma_{L}\right)-S_{12} S_{21} \Gamma_{L}} .
$$

When the load is nonreflecting,

$$
\left[\psi_{i}\right]_{\Gamma_{L}=0}=\arg \frac{S_{21}}{1-S_{11}} .
$$

If one employs the admittance matrix instead of the scattering matrix of the 2-port, the equations corresponding to (9) and (10) are

$$
\psi_{i}=\arg \frac{-Y_{21} Y_{L}}{Y_{11}\left(Y_{22}+Y_{L}\right)-Y_{12} Y_{21}},
$$

and

$$
\left[\ell_{i}\right]_{Y_{L}=1}=\arg \frac{-Y_{21}}{Y_{11}\left(Y_{22}+1\right)-Y_{12} Y_{21}},
$$

where normalized admittances are used. 
For a short-circuited phase shifter,

$$
\left[\psi_{i}\right]_{Y_{L}=0}=\arg \left(-\frac{Y_{21}}{Y_{11}}\right) .
$$

\subsection{Phase Difference Between $b_{2}$ and $a_{1}$}

One can see by inspection of the scattering equations and figure 1 that

$$
\psi_{b_{2}, a_{1}}=\arg \frac{b_{2}}{a_{1}}=\arg \frac{S_{21}}{1-S_{22} \Gamma_{L}} .
$$

When the load is nonreflecting,

$$
\left[\psi_{b_{2}, a_{1}}\right]_{\Gamma_{L}=0}=\arg S_{21}=\psi_{21}
$$

This phase shift is particularly interesting because it is simply the phase $\psi_{21}$ of the scattering coefficient $S_{21}$, a fundamental characteristic of the 2-port.

\subsection{Phase Difference between $b_{2}$ and $b_{G}$}

It is clear from (2) that the generator wave $b_{G}$ does not, in general, have the same amplitude as the wave incident upon the 2-port in arm 1 . The phase difference between $b_{2}$ and $b_{G}$ is therefore of interest and may be written

$\psi_{b_{2}, b_{G}}=\arg \frac{b_{2}}{b_{G}}=\arg \frac{S_{21}}{\left(1-S_{11} \Gamma_{G}\right)\left(1-S_{22} \Gamma_{L}\right)-S_{12} S_{21} \Gamma_{G} \Gamma_{L}}$,

or

$$
\psi_{b_{2}, b_{G}}=\arg \frac{S_{21}}{\left(1-\Gamma_{G} \Gamma_{1}\right)\left(1-S_{22} \Gamma_{L}\right)} .
$$

This phase shift depends upon the reflection coefficients of generator and load as well as upon the scattering coefficients of the 2-port. When only the load is nonreflecting,

$$
\left[\psi_{b_{2}, b_{G}}\right]_{\Gamma_{L}=0}=\arg \frac{S_{21}}{1-S_{11} \Gamma_{G}} .
$$

When only the generator is nonreflecting,

$$
\left[\psi_{b_{2}, b_{G}}\right]_{\Gamma G=0}=\arg \frac{S_{21}}{1-S_{22} \Gamma_{L}} .
$$

When both generator and load are nonreflecting,

$$
\left[\psi_{b_{2}, b_{G}}\right]_{\Gamma_{G}=\Gamma_{L}=0}=\arg S_{21}=\psi_{21} .
$$

It is observed that this is the same result as (15) and is of special interest for the same reason that (15) is of interest.

The phase difference between $b_{2}$ and $b_{G}$ may be expressed in terms of two components, (1) the phase difference between $a_{1}$ and $b_{G}$, and (2) the phase difference between $b_{2}$ and $a_{1}$ as follows.

$$
\left[\psi_{b_{2}, b_{G}}\right]=\arg \frac{b_{2}}{b_{G}}=\arg \left[\frac{b_{2}}{a_{1}} \cdot \frac{a_{1}}{b_{G}}\right]=\psi_{b_{2}, a_{1}}+\psi_{a_{1}, b_{G}} \cdot
$$

The first of these components is given by (14), and the second follows from subsequent inspection of (17).

$$
\psi_{a_{1}, b_{G}}=\arg \frac{a_{1}}{b_{G}}=\arg \frac{1}{1-\Gamma_{G} \Gamma_{1}} .
$$

Thus, one obtains

$$
\psi_{b_{2}, b_{G}}=\psi_{21}-\arg \left(1-S_{22} \Gamma_{L}\right)-\arg \left(1-\Gamma_{G} \Gamma_{1}\right) .
$$

This latter expression shows clearly how reflections from the generator and load can affect $\psi_{b_{2}}, b_{G}$.

Other phase differences such as between $b_{2}$ and $b_{1}, a_{2}$ and $a_{1}, b_{2}$, and $v_{1}$, etc., could be considered, but are perhaps of less interest than the above examples. Writing of equations for these phase differences would not be difficult, if they were desired.

\section{Differential Phase Shift}

When variable phase shifters are adjusted, one is interested in a change produced in the phase of $v_{2}$ or $b_{2}$ at the output waveguide lead of the 2-port as shown in figure 1.

For the purposes of analysis, it is convenient to regard a variable phase shifter adjustment as though one removed an initial phase shifter and substituted a final phase shifter, even though the variable phase shifter remains in the system at all times. Using front superscripts $i$ and $f$ to denote initial and final conditions, respectively, it can be shown as follows that the change in phase of $v_{2}$ produced by a given change of a variable phase shifter is the same as the corresponding change in phase of $b_{2}$. Writing the change in phase

$\Delta \psi_{v_{2}}=\arg \frac{{ }^{f} v_{2}}{{ }^{i} v_{2}}=\arg \left(\frac{{ }^{f} b_{2}+{ }^{f} a_{2}}{{ }^{i} b_{2}+{ }^{i} a_{2}}\right)=\arg \frac{{ }^{f} b_{2}}{{ }^{i} b_{2}}\left(\frac{1+{ }^{f} \Gamma_{L}}{1+{ }^{i} \Gamma_{L}}\right)$.

If there is no change in the load, ${ }^{f} \Gamma_{L}={ }^{i} \Gamma_{L}$, and

$$
\Delta \psi_{v_{2}}=\arg \frac{{ }^{f} v_{2}}{{ }^{i} v_{2}}=\arg \frac{{ }^{f} b_{2}}{{ }^{i} b_{2}}=\Delta \psi_{b_{2}}=\Delta \psi .
$$

One can now obtain an expression for the differential phase shift, using ${ }^{1}$

$$
b_{2}=\frac{S_{21} b_{G}}{\left(1-S_{11} \Gamma_{G}\right)\left(1-S_{22} \Gamma_{L}\right)-S_{12} S_{21} \Gamma_{G} \Gamma_{L}}
$$

and (25), and assuming that there is no change in $b_{G}, \Gamma_{G}$, or $\Gamma_{L}$.

\footnotetext{
1 This follows from manipulation of (2) and (3), substituting $a_{2}=b_{2} \Gamma_{L}$.
} 
The differential phase shift is

$\Delta \psi=\arg \left[\frac{{ }^{f} S_{21}}{{ }^{i} S_{21}} \cdot \frac{\left(1-{ }^{i} S_{11} \Gamma_{G}\right)\left(1-{ }^{i} S_{22} \Gamma_{L}\right)-{ }^{i} S_{12}{ }^{i} S_{21} \Gamma_{G} \Gamma_{L}}{\left(1-{ }^{f} S_{11} \Gamma_{G}\right)\left(1-{ }^{f} S_{22} \Gamma_{L}\right)-{ }^{f} S_{12}{ }^{f} S_{21} \Gamma_{G} \Gamma_{L}}\right]$.

If only the generator is nonreflecting,

$$
[\Delta \psi]_{\Gamma_{G}=0}=\arg \left(\frac{{ }^{f} S_{21}}{{ }^{i} S_{21}} \frac{1-{ }^{i} S_{22} \Gamma_{L}}{1-{ }^{f} S_{22} \Gamma_{L}}\right) .
$$

If only the load is nonreflecting,

$$
[\Delta \psi]_{\Gamma_{L}=0}=\arg \left(\frac{{ }^{f} S_{21}}{{ }^{i} S_{21}} \cdot \frac{1-{ }^{i} S_{11} \Gamma_{G}}{1-{ }^{f} S_{11} \Gamma_{G}}\right) .
$$

If both generator and load are nonreflecting,

$$
[\Delta \psi]_{\Gamma_{G}=\Gamma_{L}=0}=\arg \frac{{ }^{f} S_{21}}{{ }^{i} S_{21}}={ }^{f} \psi_{21}-{ }^{i} \psi_{21} .
$$

\section{Characteristic Phase Shift}

The foregoing equations have served to illustrate that the phase differences considered will in general depend not only upon the characteristics of the 2-port, but also upon the characteristics of the load, and in some cases also upon the characteristics of the generator.

If one is interested in a phase difference which depends only upon characteristics of the 2-port, then (5), (10), (15), and (20) can be considered. Of these, (15) and (20) are simplest. Thus it would seem desirable to select $\psi_{21}$ as one of the characteristic phase shifts of a 2-port. (The other would be $\psi_{12}$.) It would be defined ${ }^{2}$ as the phase difference between $b_{2}$ and $b_{G}$ when nonreflecting generator and load are connected to arms 1 and 2 , respectively, of the 2-port.

The differential phase shift of a 2-port in a nonreflecting system as given by (30) is then simply the differential characteristic phase difference, as defined above.

When phase differences of 2-ports are measured under different source or load conditions, different results will be expected. The discrepancies can be called mismatch errors, which can be evaluated by reference to the foregoing equations.

\section{An Ideal Phase Shifter}

The concept of an ideal phase shifter is useful for comparison purposes in evaluating the performance of actual phase shifters. Such a phase shifter would be nonreflecting ( $\left.S_{11}=S_{22}=0\right)$, and lossless, but could be nonreciprocal. Consequently

$$
S_{12} S_{21}=e^{j\left(\psi_{12}+\psi_{21}\right)} .
$$

2 This definition is in harmony with that given in IRE Standards on Antennas and Waveguides: Waveguide and waveguide component measurements, 1959 Proc. IRE 47, No. 4, 568-582.
One notes that the phase shifts of an ideal phase shifter more closely approach $\psi_{21}$, than one which is not ideal, even if generator and load reflections are present. For example,

$$
\begin{gathered}
\psi_{v}=\psi_{21}+\arg \left[\frac{1+\Gamma_{L}}{1+\Gamma_{L} e^{j\left(\psi_{21}+\psi_{12}\right)}}\right], \\
\psi_{i}=\psi_{21}+\arg \left[\frac{1-\Gamma_{L}}{1-\Gamma_{L} e^{j\left(\psi_{21}+\psi_{12}\right)}}\right], \\
\psi_{b_{2}, b_{G}}=\psi_{21}-\arg \left[1-\Gamma_{G} \Gamma_{L} e^{j\left(\psi_{21}+\psi_{12}\right)}\right],
\end{gathered}
$$

and

$$
\psi_{b_{2}, a_{1}}=\psi_{21} .
$$

The differential phase shift of an ideal phase shifter is

$$
\Delta \psi=\left({ }^{f} \psi_{21}-{ }^{i} \psi_{21}\right)+\arg \left[\frac{1-\Gamma_{G} \Gamma_{L} e^{j\left({ }^{i} \psi_{21}+{ }^{i} \psi_{12}\right)}}{1-\Gamma_{G} \Gamma_{L} e^{j\left({ }^{f} \psi_{21}+{ }^{f} \psi_{12}\right)}}\right] .
$$

It is seen that the use of an ideal phase shifter does not obviate the need for a nonreflecting system, if the characteristic phase shift is to be produced.

In an ideal situation, the output level is not changed when the phase shifter is adjusted. However, it can be seen by application of $(26)$ that the change in level of either $v_{2}$ or $b_{2}$ expressed in decibels is

$$
\begin{aligned}
& \Delta L=20 \log _{10}\left|\frac{{ }^{i} b_{2}}{{ }^{f} b_{2}}\right| \\
& =20 \log _{10}\left|\frac{S_{21}}{{ }^{i} S_{21}} \cdot \frac{\left(1-{ }^{f} S_{11} \Gamma_{G}\right)\left(1-{ }^{f} S_{22} \Gamma_{L}\right)-{ }^{f} S_{12}{ }^{f} S_{21} \Gamma_{G} \Gamma_{L}}{\left(1-{ }^{i} S_{11} \Gamma_{G}\right)\left(1-{ }^{i} S_{22} \Gamma_{L}\right)-{ }^{i} S_{12}{ }^{i} S_{21} \Gamma_{G} \Gamma_{L}}\right| .
\end{aligned}
$$

Even with an ideal phase shifter, a change in level will occur, given by

$\Delta L$ (for ideal phase shifter $)=20 \log _{10}$

$$
\left|\frac{1-\Gamma_{G} \Gamma_{L} e^{j\left({ }^{(} \psi_{21}+{ }^{f} \psi_{12}\right)}}{1-\Gamma_{G} \Gamma_{L} e^{j\left({ }^{i} \psi_{21}+{ }^{i} \psi_{12}\right)}}\right| .
$$

One can see from the above that there would be no change in level from an ideal phase shifter in a nonreflecting system $\left(\Gamma_{G}=\Gamma_{L}=0\right)$, but that such a change in level would generally occur if the phase shifter were not ideal.

\section{Conclusions}

It has been shown that one can reasonably choose either $\psi_{21}$, the argument of $S_{21}$, or $\psi_{12}$, the argument of $S_{12}$, as the characteristic phase shift of a 2-port. For a variable phase shifter, the characteristic differential phase shift $\Delta \psi$ equals the change in $\psi_{21}$ between initial and final settings.

In measuring these quantities, it is important to insert the phase shifter into nonreflecting systems 
$\left(\Gamma_{G}=\Gamma_{L}=0\right)$, and in using the phase shifter thereafter, it is no less important to duplicate these conditions. Any deviation from these conditions will result in a mismatch error, and these have been analyzed [Schafer, 1960] for some types of measurement systems.

The use of an ideal phase shifter which is lossless and nonreflecting does not obviate the need for a nonreflecting system in phase shift measurements, although the mismatch errors will usually be smaller if the phase shifter is ideal. It was shown that there will be no change in the output level of an ideal variable phase shifter when operated in a nonreflecting system, but some change can be expected if the system has reflections.

Constructive criticism and sugoestions for improving the presentation were given by David M.
Kerns of NBS and Helmut M. Altschuler of the Polytechnic Institute of Brooklyn. The encouragement given by G. E. Schafer of NBS is also gratefully acknowledged.

\section{References}

Kerns, David M. (May 1949), Basis of the application of network equations to waveguide problems, J. Res. NBS 42, No. 5, 515-540, RP1990.

Kerns, David M. (Apr. 1951), Analysis of symmetrical waveguide junctions, J. Res. NBS 46, No. 4, 267-282, RP2195.

Schafer, George E. (Nov. 1960), Mismatch errors in microwave phase shift measurements, IRE Trans. on MTT 8, No. 6, 617-622.

Sparks, Richard A. (Jan. 1963), Microwave phase measurements, Microwaves 2 , No. 1, 14-25.

(Paper 68D4-352) 